\title{
Estimation of the Deformation Field for the Left Ventricle Walls in 4-D Multislice Computerized Tomography
}

\author{
Antonio Bravo ${ }^{1}$, Rubén Medina ${ }^{2}$, \\ Gianfranco Passariello ${ }^{3}$, and Mireille Garreau ${ }^{4}$ \\ 1 Grupo de Bioingeniería, Universidad Nacional Experimental del Táchira, \\ Decanato de Investigación, San Cristóbal 5001, Venezuela \\ abravo@unet.edu.ve \\ ${ }^{2}$ Grupo de Ingeniería Biomédica, Universidad de Los Andes, Facultad de Ingeniería, \\ Mérida 5101, Venezuela \\ rmedina@ula.ve \\ ${ }^{3}$ Grupo de Bioingeniería y Biofísica Aplicada (GBBA), Universidad Simón Bolívar, \\ Sartenejas, Caracas 39000, Venezuela \\ gpass@usb.ve \\ 4 Laboratoire Traitement du Signal et de L'Image, Université de Rennes 1, \\ Rennes 35042, France \\ mireille.garreau@univ-rennes1.fr
}

\begin{abstract}
This paper describes a method for estimating the deformation field of the Left Ventricle (LV) walls from a 4-D Multi Slice Computerized Tomography (MSCT) database. The approach is composed of two stages: in the first, a 2-D non-rigid correspondence algorithm matches a set of contours on the $\mathrm{LV}$ at consecutive time instants. In the second, a 3-D curvature-based correspondence algorithm is used to optimize the initial approximate correspondence. The dense displacement field is obtained based on the optimized correspondence. Parameters like LV volume, radial contraction and torsion are estimated. The algorithm is validated on synthetic objects and tested using a 4-D MSCT database. Results are promising as the error of the displacement vectors is $2.69 \pm 1.38 \mathrm{~mm}$ using synthetic objects and, when tested in real data, local parameters extracted agree with values obtained using tagged magnetic resonance imaging.
\end{abstract}

\section{Introduction}

Heart motion studies are a sensitive indicator of heart disease, in consequence, the estimation of cardiac motion and wall deformation are important parameters for understanding the cardiac function. In particular, the evidence of reduced transmural strain and left ventricle (LV) torsion are both important indicators of myocardial ischemia 112. The detailed deformation analysis of the heart has been performed using highly invasive approaches based either on radiopaque or sonomicrometer markers implanted in the myocardium 3 [4. In these approaches, 
implantation of markers may by themselves alter the pattern of deformation [5]. More recently, non-invasive techniques based on tagged Magnetic Resonance Imaging (MRI) has been used to provide accurate estimations [67.

Several methodologies have been proposed for image analysis and for extracting parameters describing the ventricular dynamics, thus increasing the frontiers of clinical diagnoses and research on cardiovascular diseases [8]. The complete modeling of mechanical properties of cardiac structures is a problem that remains open, however, several approaches have been proposed for the description of motion and deformations of the myocardial structure based in different cardiac imaging modalities 8/910 11]. Clinical and research applications of cardiac image analysis are considerably extensive [12. However, these applications still have to overcome problems like robustness, computational complexity, $3-\mathrm{D}$ interaction and clinical validation.

Different techniques have been used for describing and quantifying the nonrigid motion of the heart. Non-rigid motion analysis is a difficult problem because the motion implies a varying shape and possibly a varying topological structure. Optical flow has been used for detecting the endocardial motion by analysis of changes in intensity in MRI images [6]. However, the displacement field is usually estimated from 2-D projections of a 3-D object, hence it is approximate. A set of physics-based models have been proposed recently, based in the space-time analysis (3-D + time) of images, which have provided a more realistic representation of cardiac chambers shape [71113. These models use geometry, kinematics, dynamics and material properties in order to model physical objects and their interactions with the physical world. The success of these approaches relies in considering a priori-knowledge about the LV, shape and motion, to predict ventricular dynamics. Simon et. al. introduced two approaches: one is based on a surface matching process [9] and the other is based on a 3-D surface/volume matching process [14. The first approach provides 3-D displacement vectors between two surfaces for consecutive time instants. The matching procedure between surfaces is performed according to an energy function composed of two terms: a data term and a regularization term. A simulated annealing is used to perform a global optimization of correspondences. The estimated displacement field can represent accurate information related to LV motion.

In this paper, a method for estimating the deformation field for the left ventricle walls from sequences of three-dimensional cardiac images is presented. An efficient non-rigid shape-based correspondence algorithm is applied to the left ventricular surfaces extracted from 4-D imaging databases. The obtained correspondence maps enable the accurate estimation of functional local wall motion indexes.

\section{Left Ventricle Geometrical Representation}

Our geometrical representation of the left ventricle is constructed from 3-D data points located in the endocardial and epicardial walls of the left ventricle. These points $\mathbf{p}(x, y, z)$ are detected from the $4-\mathrm{D}$ image dataset during the segmen- 
tation stage. Endocardial and epicardial walls are manually segmented in each slice of a 4-D MSCT database. Each segmented contour, is parameterized using a 2-D b-spline which is sampled to generate a discrete set of evenly distributed points that are considered as primary contours.

An interpolation algorithm is used with the objective of generating an isosampled set of points in three dimensions. This interpolation process is necessary because the resolution along the longitudinal axis $(\mathrm{z})$ is lower than resolution in axial plane (x-y) of the MSCT database. Each $3-\mathrm{D}$ point $\mathbf{p}(x, y, z)$ in the LV wall is located with respect to the coordinate system $\Gamma$ (Fig. 1,a). The LV wall is a surface that can be represented in the intrinsic reference system $\Pi$ known as the material coordinate system where each point $p(u, v)$ is defined in the domain $\Omega=[0,1]$. In this reference system the $u$ axis goes from the apex to the base of the LV while the $v$ axis begins in a point located in the ventricular septum and goes along the equatorial line of the shape arriving to the departing point (Fig. 1.b). Using this representation each point of the LV surface can be expressed in the coordinate system $\Gamma$ as $\mathbf{p}(x(u, v), y(u, v), z(u, v))$.

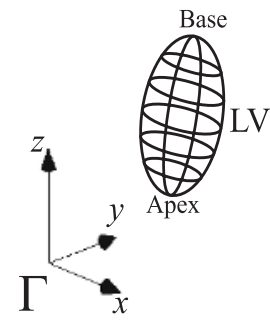

(a)

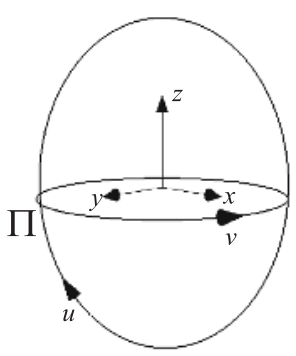

(b)

Fig. 1. Reference systems used in the geometrical representation of the LV shape. (a) $\Gamma$ coordinate reference system. (b) Material coordinate system $\Pi$.

The LV is represented as a continuous surface $s(u, v)$ using interpolation based in a set of contour points $\left(u_{k}, v_{l}\right)$ included in a given neighborhood. The resulting parametric surface is given as the convolution of the discrete samples with a B-spline 2-D interpolation kernel $h(u, v)[15]$. Such surface is represented as:

$$
s(u, v)=\sum_{k} \sum_{l} s\left(u_{k}, v_{l}\right) \cdot h\left(u-u_{k}, v-v_{l}\right),
$$

where $k, l$ define a neighborhood of $7 \times 7$ points. The continuous surface obtained is resampled at the desired sampling distance with the objective of generating new contours (secondary contours) between the given primary contours. The left ventricle endocardial and epicardial surfaces are represented by the set of original and interpolated contours. The primary and secondary contours for the endocardial wall are shown in Fig. 2 . 


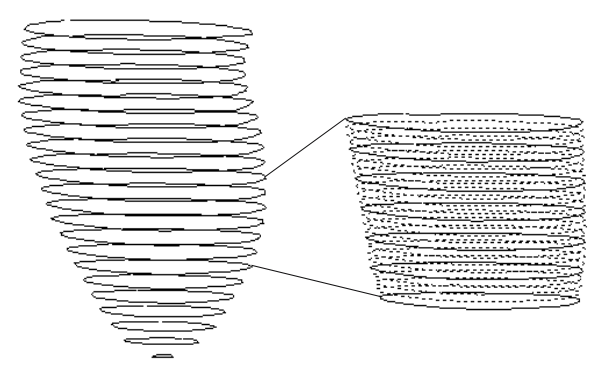

Fig. 2. Endocardial contour stacks. Left figure: the segmented contours. Rigth figure: the complete contours set (including both primary and secondary contours).

\section{Shape-Based Correspondence Algorithm}

Since the $\mathrm{LV}$ is in motion, points $\mathbf{p}(x, y, z)$ at time $t$ will move to a new position $\mathbf{p}^{\prime}\left(x^{\prime}, y^{\prime}, z^{\prime}\right)$ at time $t+1$. Thus, for non-rigid motion analysis, the problem of shape-based correspondence is to find the Euclidean transformation $Q$ that for all time instants converts the point $\mathbf{p}$ into point $\mathbf{p}^{\prime}$ :

$$
Q(\mathbf{p}, t)=\mathbf{p}^{\prime}
$$

The shape-based correspondence algorithm has two stages: the first stage corresponds to the generation of an initial estimate of correspondence based on a set of critical points [16], where the local curvature is maximum, extracted from the primary LV contours at consecutive time instants. Then, in the second stage the algorithm optimizes the initial correspondence in the $3-\mathrm{D}$ space using both primary and secondary contours.

\subsection{2-D Non-rigid Correspondence Algorithm}

In the first stage we use a 2-D approach based on tracking a set of Critical Points in the primary contours of the LV geometrical representation, using the non-rigid correspondence algorithm proposed by Hill et al. [17. This algorithm transforms a discretized contour $\mathbf{A}=\left\{\mathbf{A}_{i} ; 1 \leq i \leq n_{A}\right\}$ (a primary LV contour at time $t$ ), onto some other contour $\mathbf{B}=\left\{\mathbf{B}_{i} ; 1 \leq i \leq n_{B}\right\}$ (a primary LV contour at time $t+1$ ), where $n_{A}$ and $n_{B}$ are the number of points in contours $\mathbf{A}$ and $\mathbf{B}$ respectively. The algorithm produces two new shapes $\mathbf{A}^{\prime}=\left\{\mathbf{A}_{\alpha_{i}} ; 1 \leq\right.$ $\left.i \leq n_{\Phi}\right\}$ and $\mathbf{B}^{\prime}=\left\{\mathbf{B}_{\beta_{i}} ; 1 \leq i \leq n_{\Phi}\right\}$ that are in correspondence and represent sparse subpolygons of $\mathbf{A}$ and $\mathbf{B}$ respectively. The sparseness is related to the fact that each contour has less points than the original thus increasing the distance between each pair of points. The correspondence is defined by a set of ordered pairs $\Phi=\left\{\phi_{i}=\left(\alpha_{i}, \beta_{i}\right) ; 1 \leq i \leq n_{\Phi}\right\}$, where integer values $\left\{\alpha_{i}\right\}$ index the points of $\mathbf{A}$ and $\left\{\beta_{i}\right\}$ index points of $\mathbf{B}$ that are in correspondence.

The Hill's non-rigid correspondence algorithm comprises three parts: 
1. Generation of shape approximations to both $\mathbf{A}$ and $\mathbf{B},\left(\mathbf{A}^{\prime \prime}\right.$ and $\mathbf{B}^{\prime \prime}$ respectively). These approximate shapes only contain $n_{A^{\prime \prime}}$ critical points of $\mathbf{A}$ and $n_{B^{\prime \prime}}$ critical points of $\mathbf{B}$ (usually $n_{A^{\prime \prime}} \neq n_{B^{\prime \prime}}$ ). In this stage no correspondence is established. The critical point detection (CPD) algorithm described by Zhu and Chirlian [18 is used. This CPD algorithm does not require explicit curvature estimation, the algorithm is also reproducible, reliable, invariant, and symmetric.

2. Generation of an initial correspondence between $\mathbf{A}^{\prime}$ and $\mathbf{B}^{\prime}$. The pathmatching algorithm is used. A reference of correspondence is established $\left(\alpha_{0}=1, \beta_{i}=i\right)$. The path-length spacing of the points defining $\mathbf{A}^{\prime \prime}$ (with respect to $\mathbf{A}_{1}$ ) are projected onto $\mathbf{B}$ (with respect to $\mathbf{B}_{i}$ ) and also the pathlength spacing of the points defining $\mathbf{B}^{\prime \prime}$ (with respect to $\mathbf{B}_{i}$ ) are projected onto $\mathbf{A}$ (with respect to $\left.\mathbf{A}_{1}\right)$. This process generates $\left[\left(n_{A^{\prime \prime}}+n_{B^{\prime \prime}}\right) * n_{B}\right]$ possible sets of correspondences. The best correspondence will be reached when the pixel $\mathbf{B}_{i}$ that matches the pixel $\mathbf{A}_{1}$ is identified by minimizing the following cost function:

$$
\min E_{i}^{2}=\sum_{j=1}^{n_{A^{\prime \prime}}+n_{B^{\prime \prime}}}\left\|\mathbf{A}_{\alpha_{j}}-Q\left(\mathbf{B}_{\beta_{j}}\right)\right\|^{2},
$$

where $Q$ represent the Euclidean transformation $Q(\mathbf{p})=s \mathbf{R} \mathbf{p}+\mathbf{t}, s$ is a scale factor, $\mathbf{R}$ is a rotation matrix, and $\mathbf{t}$ is a traslation. This patch-matching algorithm produces a set of correspondences $\Phi=\left\{\phi_{i} ; 1 \leq i \leq\left(n_{A^{\prime \prime}}+n_{B^{\prime \prime}}\right)\right\}$. For each pair of correspondence points $\left(\mathbf{A}_{\alpha_{i}}, \mathbf{B}_{\beta_{i}}\right)$, the value $T_{i}$ is calculated:

$$
T_{i}=\max \left(\operatorname{Area}\left(\mathbf{A}_{\alpha_{i-1}}, \mathbf{A}_{\alpha_{i}}, \mathbf{A}_{\alpha_{i+1}}\right), \operatorname{Area}\left(\mathbf{B}_{\alpha_{i-1}}, \mathbf{B}_{\alpha_{i}}, \mathbf{B}_{\alpha_{i+1}}\right)\right),
$$

where $\operatorname{Area}(\cdot)$ computes the area of a triangle whose vertices are three consecutive points (for instance, $\mathbf{A}_{\alpha_{i-1}}, \mathbf{A}_{\alpha_{i}}, \mathbf{A}_{\alpha_{i+1}}$ ). The $\phi_{i}$ for which $T_{i}$ is minimum is deleted repeatedly until $n_{\Phi}=\left(n_{A^{\prime \prime}}+n_{B^{\prime \prime}}\right) / 2$ correspondences are obtained.

3. An iterative local optimization scheme is used to refine the initial set of correspondence by minimizing a cost function. The cost function $E$ is expressed as:

$$
E=\lambda E_{S}+(1-\lambda) E_{R},
$$

where the first term $E_{S}$ measures the difference in shape between $\mathbf{A}^{\prime}$ and its corresponding polygon $\mathbf{B}^{\prime}$, represented as a mean distance error. The second term $E_{R}$ ensures that the manner in which $\mathbf{A}^{\prime}$ differs from $\mathbf{A}$ is as similar as possible to the manner in which $\mathbf{B}^{\prime}$ differs from $\mathbf{B}$ and it is expressed as a mean distance error. The parameter $\lambda$ expresses the relative contribution of each of the terms included in the cost function and their value is taken based in the experimental results obtained by Hill et al. [17.

The method for non-rigid correspondence proposed by Hill, is used in the framework for automatic landmark identification in a set of 2-D shapes representing an object. Within this framework the objective is to obtain a mean 
shape that represents the set of 2-D shapes based on the information provided by the method of non-rigid correspondence. With this purpose, Hill et al. 17] proposed an algorithm that constructs a binary tree whose root is the mean shape. In our application the goal is to establish the correspondence between primary contours, at consecutive time instants, describing the 4-D LV shape. Thus, the correspondence during the entire cardiac cycle could be modeled as a transformation defined by the composition of several time-consecutive transformations as the LV motion is small and varies between two consecutive time instants [7. The algorithm proposed by Hill et al. for constructing the binary tree is modified. In this application the construction of the matrix of correspondence values, considers only pairs of contours that are consecutive in time. The rest of steps of the algorithm are followed to arrive to the mean representative shape located in the root of the tree. In the mean shape the critical point detection algorithm is applied and then, these critical points are projected back along the tree towards the leaves to arrive to the optimal correspondence between primary contours.

\subsection{Curvature Based Correspondence Optimization}

The non-rigid correspondence algorithm described in the previous section, gives a set of correspondences for all primary contours extracted from the MSCT database. If $\mathbf{p}_{1}\left(x_{1}, y_{1}, z_{1}\right)$ is a point on a primary contour of the LV surface $s_{1}$ at time $t_{1}$ and $\mathbf{p}_{2}\left(x_{2}, y_{2}, z_{2}\right)$ its corresponding point on the LV surface $s_{2}$ at time $t_{2}$, then the displacement vector for the point $\mathbf{p}_{1}, \mathbf{v}\left(\mathbf{p}_{1}\right)$ is given by:

$$
\mathbf{v}\left(\mathbf{p}_{1}\right)=\mathbf{p}_{2}-\mathbf{p}_{1}
$$

Since all points of a primary contour are on the same axial plane, the non-rigid correspondence method does not consider the through-plane component of the 3-D motion field. Considering that the motion of the heart is sufficiently small between consecutive 3-D images (10-18 images acquired for a cardiac cycle) [7], we can track the evolution of curvature in selected regions or patches of the LV geometrical representation [13. We use the shape-based tracking algorithm proposed by Shi 19. This algorithm tries to match points on successive surfaces using a shape similarity metric. Such a metric $(\epsilon)$ is based on the difference in principal curvatures $k_{1}$ and $k_{2}$.

$$
\epsilon=\frac{\left[k_{1}\left(\mathbf{p}_{1}\right)-k_{1}\left(\mathbf{p}_{2}\right)\right]^{2}+\left[k_{2}\left(\mathbf{p}_{1}\right)-k_{2}\left(\mathbf{p}_{2}\right)\right]^{2}}{2} .
$$

The shape-based tracking verifies if a point near $\mathbf{p}_{2}\left(\hat{\mathbf{p}}_{2}\right)$ exists, where the shape similarity metric achieves a minimum. The set of neighbor points $\left\{\hat{\mathbf{p}}_{2, \mathrm{i}}\right\}$, consist of all points on $s_{2}$ that have a distance less than a threshold $\delta$ from $\mathbf{p}_{2}$ on $s_{2}$. The euclidean distance metric is used and $\delta$ is fixed at $0.3125 \mathrm{~mm}$. The geometrical representation of the $\mathrm{LV} s_{2}$ at time $t_{2}$, considers the primary and secondary contours. The shape similarity metric (7), measures the difference between the principal curvatures of a single point $\mathbf{p}_{1}$ on $s_{1}$ and the neighbor points to $\mathbf{p}_{2}$ on $s_{2}$. Then the point $\hat{\mathbf{p}}_{2} \in\left\{\hat{\mathbf{p}}_{2, \mathrm{i}}\right\}$ which has the minimum value 


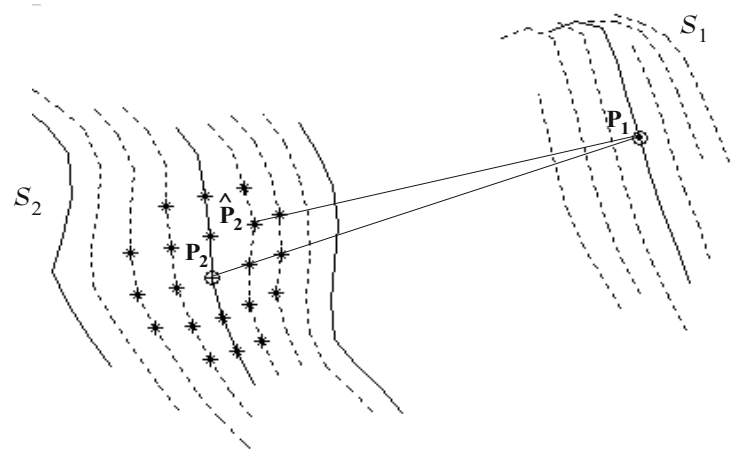

Fig. 3. Search of the new point in correspondence

of $\epsilon$ (most similar shape-properties to $\mathbf{p}_{1}$ ) is selected as the new correspondence point. This is illustrated in Fig. 3. Principal curvatures are estimated using the method proposed by Sander and Zucker [20].

\section{Results}

\subsection{Validation Using Synthetic Data}

An ellipsoidal model is used for validation of the algorithm of motion estimation. With this purpose a 3-D ellipsoidal model is deformed considering five types of motion: translation, radial contraction, longitudinal shortening and torsion. An algorithm based on Free Form Deformations (FFD) 2122] is used for deforming the initial shape leading to a deformed shape according to a predefined set of motion parameters. In each deformation stage using the FFD algorithm, the points $\mathbf{p}_{1}\left(x_{1}, y_{1}, z_{1}\right)$ before the transformation, and $\mathbf{p}_{2}\left(x_{2}, y_{2}, z_{2}\right)$ after the transformation are known, thus the displacement field is accurately obtained using (6). This motion field is compared with the motion field obtained using the algorithm of motion estimation. In this case the distance between vector endpoints is considered as a measure of errors in the motion estimation. The error obtained (mean \pm standard deviation) using a population of 42 deformations is $2.69 \pm 1.38 \mathrm{~mm}$ with a minimum value of $1.06 \mathrm{~mm}$ and a maximum value of $5.54 \mathrm{~mm}$. This is close to the value of $2.00 \mathrm{~mm}$ obtained by Chandrashekara et al. 7] using 2-D slices in tagged MRI.

\subsection{Results on Real Data}

The motion estimation algoritm is also tested using real data corresponding to a 4-D MSCT human heart database. In this database the left ventricle endocardial and epicardial walls are extracted using manual segmentation from 18 3-D images corresponding to time instants evenly spaced along the cardiac cycle. In these images, the portion above the mitral valve is excluded because the goal, 

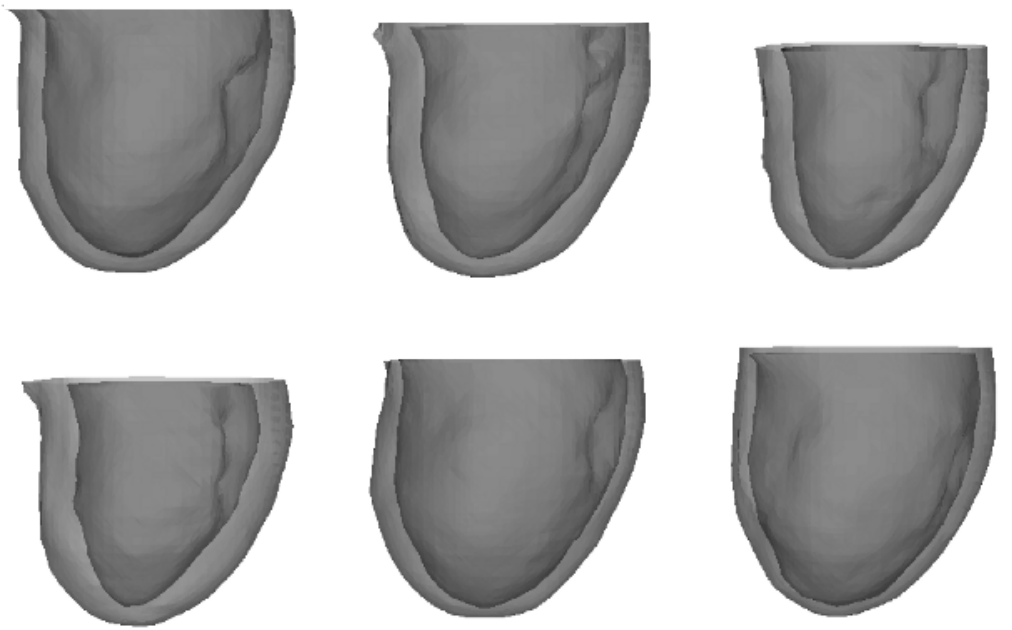

Fig. 4. Shape of the left ventricle for several time instants of the cardiac cycle

in this research, is to study only the Left Ventricular motion. Figure 4 shows the resulting LV shape for six time instants of the cardiac cycle. The longitudinal shortening that is one of the components of ventricular motion is apparent in the images shown. The motion estimation algorithm is applied to the entire 4-D sequence considering the shape correspondence algorithm and the optimization stage based in curvatures. As a result the estimated motion field for the MSCT database is obtained. Figure 5 shows a plot of the motion vectors for the endocardial wall considering three time instants of the cardiac cycle corresponding to end-diastole, $50 \%$ of diastole and end systole. Observe that the magnitude of motion increases as the time approaches the end-systole instant, this is due to the endocardial contraction. The torsion is also apparent in the end-systole instant. Estimation of the motion field for the LV endocardial wall

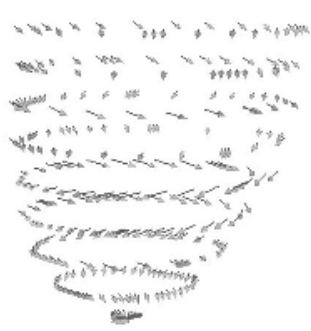

(a)

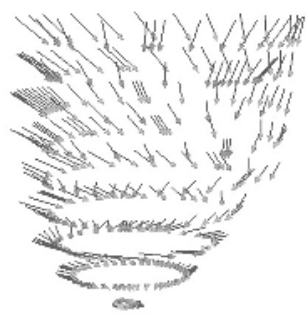

(b)

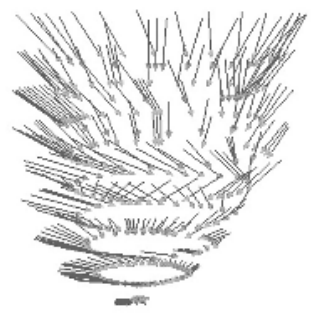

(c)

Fig. 5. Displacement vectors plot. (a) End-diastole. (b) $50 \%$ diastole. (c) End-systole 


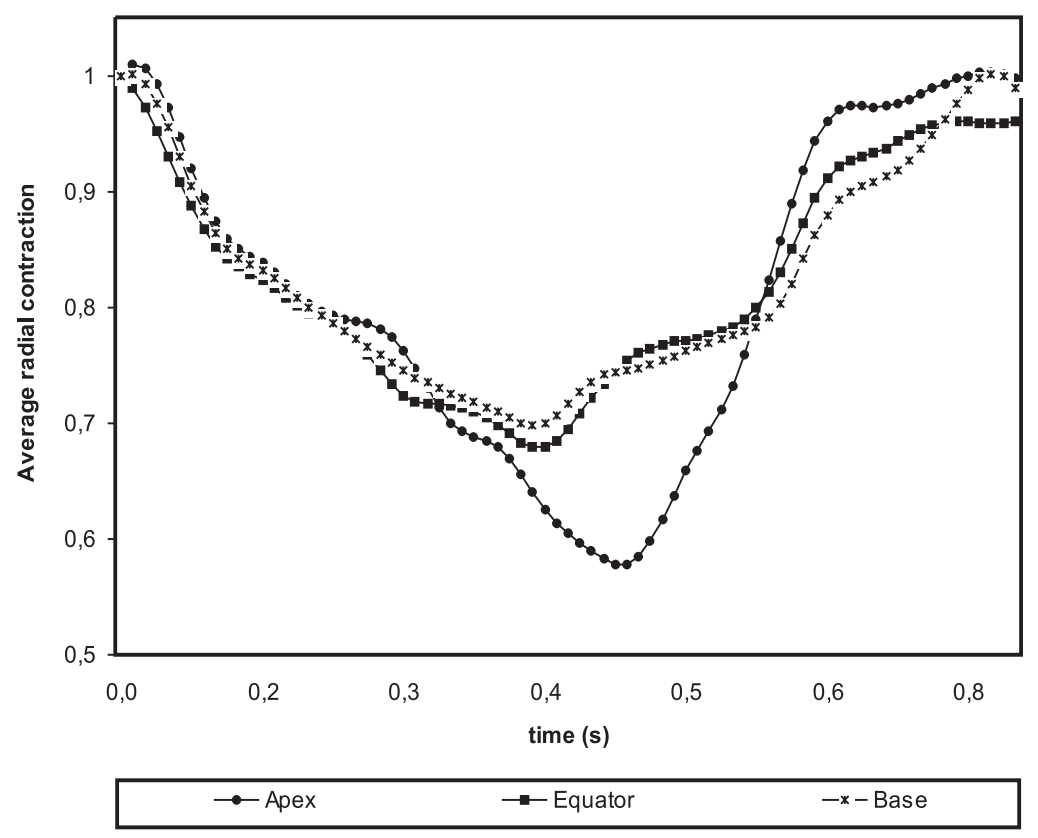

Fig. 6. Average radial contraction of the endocardial wall

enables the calculation of several local mechanical parameters associated with the ventricular motion. These parameters are the average radial contraction and torsion. The average radial contraction represents the average of radial lengths for the endocardial wall in a given axial plane. Figure 6 shows the endocardial LV radial contraction over three axial planes as a function of time. The apex plane is located $10 \mathrm{~mm}$ above the actual endocardial apex, the equator axial plane is located in the middle of the distance between the actual apex and the base. The base plane is located $10 \mathrm{~mm}$ below the actual base. The average radial contraction index is expressed normalized with respect to the value obtained in end-diastole. Results obtained for the average radial contraction are alike to the values obtained in other research works performed in tagged magnetic resonance imaging like these reported by Allouche et al. [10] and Sermesant 23] or in 3-D echocardiography as reported by Gérard et al. 11. Using the proposed method the average radial contraction in the endocardium varies between $30.20 \%$ and 42.22\% while Allouche et al. [10] obtained values between $28 \%$ and $38 \%$ using tagged MRI. Figure 7 shows the torsion value obtained over the entire cardiac cycle for the apex, equator and base plane. The torsion angle is defined as the angle between a radial line traced joining the gravity center of the slice and a endocardial contour point at time $t$ and the radial line joining the gravity center and the corresponding endocardial contour point for the $t+1$ time instant. In this case, the torsion value is higher in the apex than in the base of the endocar- 


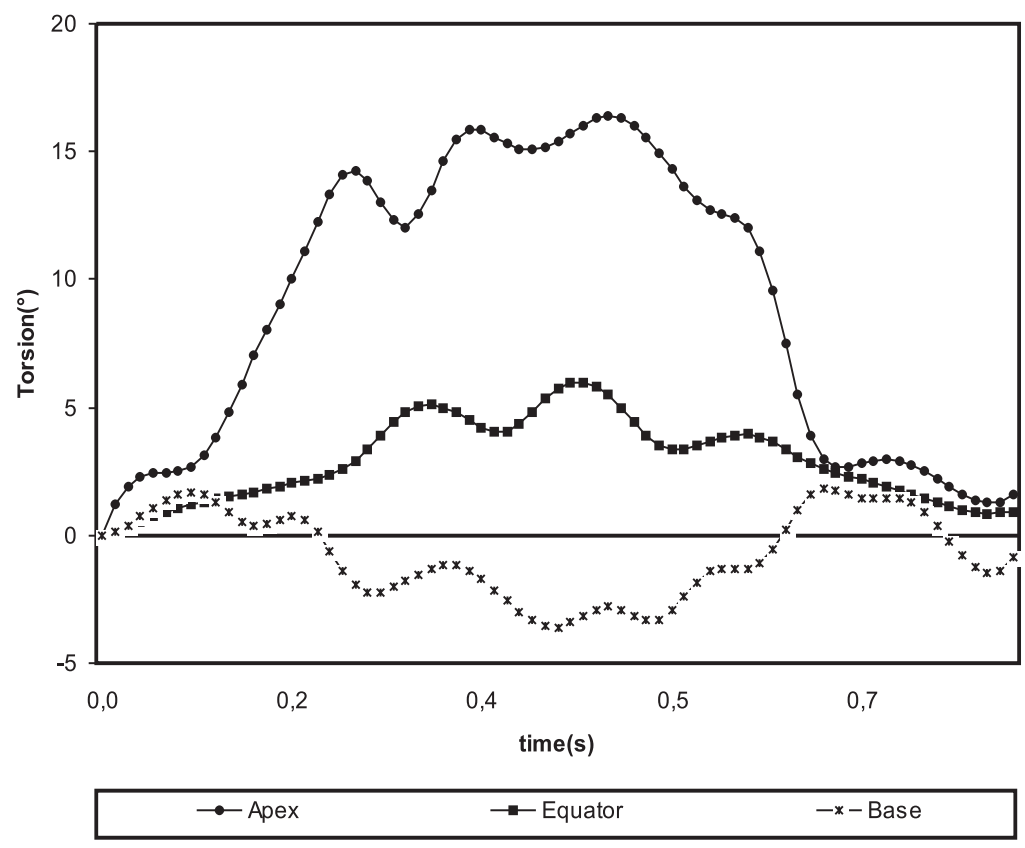

Fig. 7. Endocardial wall torsion

dial wall. Additionally, the torsion angle is opposite between the base and the apex. These features of LV motion are considered normal in healthy subjects [24. The torsion obtained is also alike to the results reported by Allouche et al. [10], Sermesant [23] and by Gérard et al. 11]. Using the proposed method the minimal torsion value $\left(-3.60^{\circ}\right)$ occurs at the base and the maximal torsion value $\left(16.40^{\circ}\right)$ occurs at the apex while Allouche et al. [10], using tagged MRI, obtained a minimum value at the base of $-2.5^{\circ}$ while the maximum value of $12^{\circ}$ is obtained at the apex.

\section{Conclusions}

A method for the quantification of LV deformations have been presented. The approach presented considers local geometrical features based in curvature analysis and the assumption that the LV motion is smooth during the entire cardiac cycle. It uses local information of the shapes with the objective of providing an accurate correspondence between consecutive time instants.

Validation of the method considering synthetic data provides low error values for the distances of the vector endpoints of the estimated motion field. Test on real data shows that LV estimated motion during the cardiac cycle is consistent with the LV motion reported by the literature concerning normal subjects. The estimated displacement field reproduces the contraction and relaxation of the 
normal LV accurately. Additionally, the method enables the calculation of several global and local parameters that are useful for the assessment of cardiac motion like the volume, the average radial contraction and the torsion index. Results obtained on real data agree with other research works based on tagged MRI.

A more complete clinical validation including healthy subjects as well as subjects cursing illnesses affecting the cardiac motion is necessary. The validation should also compare results using other modalities like tagged MRI. Future research will consider the incorporation of motion information extracted from the gray level information of the MSCT database.

\section{Acknowledgment}

The authors would like to thank the Investigation Dean's Office of Universidad Nacional Experimental del Táchira, the Parallel Computer Center (CeCalCULA) and CDCHT from Universidad de Los Andes, and the University Sector Planning Office (OPSU) through its Program Alma Mater for its support of this project. Authors would also like to thank Hervé Le Breton and Dominique Boulmier from the Centre Cardio-Pneumologique in Rennes, France for providing the human MSCT database.

\section{References}

1. L. Opie. Mechanics of cardiac contraction and relaxation. In E. Braunwald, D. Zipes, and P. Libby, editors, Heart Disease: A Textbook of Cardiovascular Medicine, pages 443-478. W. B. Saunders, 6 edition, 2001.

2. T. Arts, S. Meerbaum, and R. Reneman. Torsion of the left ventricle during the ejection phase in the intact dog. Cardiovasc. Res., 18:183, 1984.

3. N. Ingels, G. Daughters, E. Stinson, and E. Alderman. Evaluation of methods for quantitating left ventricular segmental wall motion in man using myocardial markers as a standard. Circ., 61(5):966-972, 1980.

4. F Villarreal, L. Waldman, and W. Lew. Technique for measuring regional twodimensional finite strains in canine left ventricle. Circ. Res., 62(4):711-721, 1988.

5. T. Fenton, J. Cherry, and G. Klassen. Transmural myocardial deformation in the canine left ventricle wall. Am. J. Physiol. Heart Circ. Physiol., 235(4):H523-H530, 1978.

6. L. Dougherty, J. C. Asmuth, A. S. Blom, L. Axel, and R. Kumar. Validation of an optical flow method for tag displacement estimation. IEEE Trans. Med. Imag., 18(4):359-363, 1999.

7. R. Chandrashekara, R. Mohiaddin, and D. Rueckert. Analysis of 3-D myocardial motion in tagged MR images using nonrigid image registration. IEEE Trans. Med. Imag., 23(10):1245-1250, 2004.

8. A. J. Frangi, D. Rueckert, and J. S. Duncan. Three-dimensional cardiovascular image analysis. IEEE Trans. Med. Imag., 21(9):1005-1010, 2002.

9. A. Simon, M. Garreau, D. Boulmier, J.-L. Coatrieux, and H. Le Breton. Cardiac motion extraction using 3D surface matching in multislice computed tomography. In Medical Image Computing and Computer-Assisted Intervention MICCAI 2004, number 3217 in Lecture Notes in Computer Science (LNCS), pages 1057-1059, Springer, 2004. 
10. C. Allouche, S. Makram, N. Ayache, and H. Delingette. A new kinetic modeling scheme for the human left ventricle wall motion with MR-tagging imaging. In Functional Imaging and Modeling of the Heart (FIMH01), number 2230 in Lecture Notes in Computer Science (LNCS), pages 61-68, Springer, 2001.

11. O. Gérard, A. C. Billon, J.-M. Rouet, M. Jacob, M. Fradkin, and C. Allouche. Efficient model-based quantification of left ventricular function in 3-D echocardiography. IEEE Trans. Med. Imag., 21(9):1059-1068, 2002.

12. A. J. Frangi, W. J. Niessen, and M. A. Viergever. Three-dimensional modeling for functional analysis of cardiac images: A review. IEEE Trans. Med. Imag., 20(1):2-25, 2001.

13. X. Papademetris, A. J. Sinusas, D. P. Dione, R. T. Constable, and J. S. Duncan. Estimation of 3-D left ventricular deformation from medical images using biomechanical models. IEEE Trans. Med. Imag., 21(7):786-800, 2002.

14. A. Simon, M. Garreau, D. Boulmier, J.-L. Coatrieux, and H. Le Breton. A surface/volume matching process using a markov random field model for cardiac motion extraction in MSCT imaging. In Functional Imaging and Modeling of the Heart (FIMH05), Lecture Notes in Computer Science (LNCS), Barcelona, Spain, June 2005. Accepted for publication.

15. T. Lehmann, C. Gönner, and K. Spitzer. Survey: Interpolation methods in medical image processing. IEEE Trans. Med. Imag., 18(11):1049-1073, 1999.

16. A. Yuille and T. Poggio. Scaling theorems for zero crossings. IEEE Trans. Pattern Anal. Machine Intell., 8(1):15-25, 1986.

17. A. Hill, C. Taylor, and A. Brett. A framework for automatic landmark identification using a new method of nonrigid correspondence. IEEE Trans. Pattern Anal. Machine Intell., 22(3):241-251, 2000.

18. P. Zhu and P. Chirlian. On critical point detection of digital shapes. IEEE Trans. Pattern Anal. Machine Intell., 17(8):737-748, 1995.

19. P. Shi. Image Analysis of $3 D$ Cardiac Motion Using Physical and Geometrical Models. PhD thesis, Yale University, May 1996.

20. P. Sander and S. Zucker. Inferring surface trace and differential structure from 3-D images. IEEE Trans. Pattern Anal. Machine Intell., 12(9):833-854, 1990.

21. T. Sederberg and S. Parry. Free-form deformation of solid geometric models. Comput. Graph., 20(4):537-541, 1986.

22. A. Bravo, R. Medina, G. Passariello, and M. Garreau. Deformable parametric model for left ventricle wall motion simulation. In Proceedings of the 14th IASTED International Conference on Applied Simulation and Modelling ASM 2005, ACTA Press, pages 24-29, Benalmádena, Spain, june 2005.

23. M. Sermesant. Modéle électromécanique du cœur pour l'analyse d'image et la simulation. $\mathrm{PhD}$ thesis, Université de Nice Sophia-Antipolis, Institut National de Recherche en Informatique et Automatique (INRIA), France, 2003.

24. A. Sniderman, D. Marpole, and E. Fallen. Regional contraction patterns in the normal and ischemic left ventricle of man. Amer. J. Cardiol., 31(4):484-489, 1973. 